Electronic version of an article published as Journal of Porphyrins and Phthalocyanines, vol. 23, no. 07/08, 2019, p. 916-929, DOI: 10.1142/S1088424619500809 @ World Scientific Publishing Company https://www.worldscientific.com/doi/pdf/10.1142/S1088424619500809 


\title{
Supramolecular block copolymers incorporating chiral and achiral chromophores for the bottom-up assembly of nanomaterials
}

\author{
Marta Riba-Moliner ${ }^{\mathrm{a}}$, Cristina Oliveras-González ${ }^{\mathrm{b}}$, David B. Amabilino ${ }^{c}$ \\ and Arántzazu González-Campo ${ }^{a *}$ \\ anstitut de Ciència de Materials de Barcelona (ICMAB-CSIC), Campus Universitari, 08193 Bellaterra, \\ Catalonia, Spain \\ ${ }^{\mathrm{b}}$ Université d'Angers, CNRS Laboratoire MOLTECH-Anjou, UMR 6200, UFR Science, Bât. K, 2 Bd. \\ Lavoisier, 49045 Angers, France \\ 'School of Chemistry, The University of Nottingham, University Park, Nottingham, NG7 2RD, UK
}

\begin{abstract}
The coordination of the chiral metalloporphyrin ([5,10,15,20-[4- $(R, R, R, R)-2-N-$ octadecylamidoethyloxiphenyl]porphyrin] zinc (II)) and an achiral homologue to an amphiphilic block copolymer of poly(styrene- $b$-4-vinyl pyridine) (PS- $b$-P4VP) have been studied in solution and as cast material. The resulting chiral dye-polymer hybrid material has been accomplished via axial coordination between the zinc (II) metal ion in the core of the porphyrin ring and the pyridyl units of the block-copolymer in a non-coordinative solvent. The supramolecular organization and possible chirality transfer to the hybrid material have been studied in solution by UV-vis absorption spectroscopy, fluorescence spectroscopy, Nuclear Magnetic Resonance and Circular Dichroism. The morphology of the chiral and achiral doped polymers has been studied in solid state by Transmission Electron Microscopy and Atomic Force Microscopy. We show that the nanostructures formed depend greatly upon the nature of the side-chains on the porphyrins, where a chiral group leads to a very homogeneous phase-separated material, perhaps indicating that chiral side groups are useful for the preparation of this type of supramolecular hybrid.
\end{abstract}

KEYWORDS: poly(styrene- $b$-4-vinyl pyridine) copolymer, chiral metalloporphyrin, doped film, micelles, hybrid material, axial coordination.

\section{INTRODUCTION}

Synthetic supramolecular chemistry has significant potential impact in the polymer field because the properties of new hybrid materials are unique, thanks to their structures and functionalities [1]. For instance, polymer aggregation can be controlled by choosing recognition of strategic elements in macromolecular chains [2]. The

*Correspondence to: Arántzazu González-Campo, Institut de Ciència de Materials de Barcelona (ICMAB-CSIC), Campus Universitari, 08193 Bellaterra, Catalonia, Spain, tel.: +34 935801856, e-mail: agonzalez@icmab.es. aim of the present work is to combine these features with those of $\pi$-functional units. Porphyrins are versatile components in supramolecular systems [3-5], and they have been studied widely due to their optical and electronic properties which make them suitable for a great variety of interests such as nanoelectronic systems [6], lightharvesting arrays [7] or dye-sensitizing chromophores in photovoltaic devices [8]. Their structural and physical properties are tuned by modifying either the side groups linked at the meso-position of the porphyrin ring or by chemical changes in the core, with the introduction of a metal ion in the chelating macrocycle being the simplest modification possible $[9,10]$. Free-base porphyrins and 
metalloporphyrins can self-assemble by non-covalent interactions [11] (such as hydrogen-bonding, $\pi-\pi$ interactions or Van der Waals forces), but the coordination of the metal ion with an exo-facing ligand can also drive the self-assembly of the chromophores [12]. Recently, the ability of these metalloporphyrins to coordinate axial groups has led to a number of novel supramolecular constructs that show interesting dynamic properties in the motion of the ring [13-15].

At the same time, organic thin films based on amphiphilic block copolymers (BCPs) have been deeply studied due to the tunability of the different structural patterns adopted between the immiscible blocks at the interface [16-19]. The volume fraction of each block and the solvent used to dissolve/disperse them are the key factors to control the type of organization adopted by the systems [20-24]. The BCP used here is poly(styrene- $b$-4-vinyl pyridine) (PS- $b$-P4VP), an amphiphilic macromolecule that contains well-defined microstructures thanks to the incompatibility of the poly(styrene) (PS) and poly(4-vinyl pyridine) (P4VP) blocks with one another, its malleability, $\mathrm{pH}$-responsiveness and the potential reactivity, and complex-forming ability of the P4VP block combined with the stiffness of the PS part. The P4VP block is selectively solubilized by polar protic solvents, except in water, where it swells [25]. Conversely, the PS block is selectively soluble in non-polar solvents. There are some nonselective solvents that dissolve PS- $b$-P4VP completely, like chloroform and tetrahydrofuran and the corresponding polar-non-polar solvent mixtures. These 'solubility rules' depend on the molar fraction of each block and the degree of polymerization. Moreover, the amphiphilic character of the BCP allows a good phase separation that can be adjusted and to some degree induced, by controlling the molar fraction and the solvent in which it is partially or totally solubilized. The variety of materials that can be achieved such as membranes, micelles and nanostructured thin films, among others, with totally different final properties [26-30], together with the use of the pyridine ring as an anchoring point have made this BCP applicable for a wide range of systems [31-33].

Here, the opportunities offered by the combination of PS- $b$-P4VP and a chiral zinc(II)-metalloporphyrin (Zn$(R, R, R, R)-\mathbf{1})$ are explored with the ultimate aim of obtaining dye-polymer hybrid materials via axial metal ion coordination for potential applications in optoelectronics $[33,34]$. The zinc (II) metal ion located in the core of the porphyrin ring will provide the possibility of coordination by the pyridyl units of the BCP, resulting in supramolecular self-assembled systems [35]. For this purpose, chiral and achiral metalloporphyrins $\mathrm{Zn}-(R, R, R, R)-\mathbf{1}$ and Zn-2 were used (Fig. 1) [36-38]. The determination of the coordination between the PS- $b$-P4VP and the Zn porphyrins, as well as the possible chirality transfer to the supramolecular organization of the BCP with the porphyrinic derivative compounds, will be studied in solution and in solid state by UV-vis absorption spectroscopy (UV-vis), Fluorescence spectroscopy and Circular Dichroism (CD) spectroscopy. Moreover, morphological studies of the superstructure formed by the supramolecular hybrid will be analyzed by Transmission Electron Microscopy (TEM) and Atomic Force Microscopy (AFM).

\section{EXPERIMENTAL}

\section{General methods}

Commercially available reagents and solvents. Styrene $\geq 99 \%$, 4-vinyl pyridine (containing 100 ppm hydroquinone as inhibitor), $\alpha$-methystyrene $99 \%$ (containing

Fig. 1. Representation of the coordination of the Zn porphyrins and the PS- $b$-P4VP after their axial coordination 
$15 \mathrm{ppm}$ p-tert-butylcatechol as inhibitor), the initiator sec-butyllithium (1.4 M in cyclohexane) and methanol (anhydrous, 99.8\%) were purchased to Sigma-Aldrich. THF was HPLC degree from Teknokroma.

Measurements. UV-vis absorbance spectroscopy was measured using a Varian Cary 5000 UV-vis spectrophotometer, in solution, with standardized quartz cuvettes of $1 \mathrm{~cm}$ of optical path. In solid experiments, standardized quartz slides were used. Quartz surfaces were cleaned with piranha solution $\left(\mathrm{H}_{2} \mathrm{SO}_{4} / \mathrm{H}_{2} \mathrm{O}_{2}(30 \%) ; 3: 1_{\mathrm{v} / \mathrm{v}}\right)$ for $30 \mathrm{~min}$ prior to use. Nuclear Magnetic Resonance (NMR) spectra were recorded in a Bruker AVANCEII 300 and Bruker Avance DRX 300 spectrometer. MatrixAssisted Laser Desorption-Ionization Mass Spectrometry (MALDI-TOF) measurements were performed with an Ultraflex (TOF/TOF) spectrometer. All Fourier Transform Infrared Spectroscopy (FTIR) measurements were recorded using a Perkin Elmer (Spectrum one) spectromenter. The samples were of solid porphyrins (recovered after evaporation of solvent in a non-controlled precipitated way) and were performed in attenuated total reflection (ATR) mode. Porphyrins were measured on a universal ATR (UATR) plate. A Jasco J-275 spectropolarimeter was used for Circular Dichroism (CD) spectra measurements. A Peltier-temperature programmer for thermosetting the samples was used to cool the solutions. The samples were transferred to a standardized quartz cuvette to be analyzed. When the measured temperature was reached and stabilized, spectra were recorded. Fluorescence spectroscopy measurements were performed using Horiba-Jobin-Yvon SPEX Nanolog-TM and Cary eclipse spectrofluorimeters. All the samples were prepared by diluting a solid sample of the porphyrin and $\mathrm{BCP}$ in the corresponding amount of solvent. Transmission Electron Microscopy (TEM) images were acquired at $120 \mathrm{kV}$. TEM supports used were holey carbon coated copper grids (200 lines/inch). Only samples containing pure PS- $b$-P4VP were selectively stained with iodine vapors, and the process occurred by placing the samples in a sealed container in an iodine vapor atmosphere for $1 \mathrm{~h}$. $5 \mathrm{kV}$, a working distance of $10 \mathrm{~mm}$, a spot of 3.5 and high vacuum were the conditions used to acquire Scanning Electron Microscopy (SEM) images. Energydispersive X-ray spectroscopy (EDAX) was assembled to the SEM microscope with $15 \mathrm{kV}$ and analyzing times of $\sim 2500 \mathrm{~s}$ were the fixed conditions to collect information.

\section{Synthesis}

Polymerization of PS- $b$-P4VP. PS- $b$-P4VP (molar ratio PS:P4VP 1:4) was synthesized following the methodology described by Varshney et al. [39]. The exact procedure, amounts and volumes employed in the reaction are detailed in the Supporting Information.

Synthesis of porphyrins Zn-(R,R,R,R)-1 and Zn-2. $[5,10,15,20$-[4- $(R, R, R, R)-2-N$-octadecylamidoethyloxiphenyl]porphyrin] zinc (II) (Zn-(R,R,R,R)-1) and
[5,10,15,20-[4- $N$-octadecylacetamidophenoxy]porphyrin] zinc (II) $(\mathrm{Zn}-2)$ were prepared according to literature procedures [37, 38].

Solid samples. A dome was used to reach vacuum conditions to obtain solid samples for TEM analyses of the phase segregation and micellar aggregation.

Polymeric doped micelles. Solvent exchange methodology: $3 \mathrm{mg}$ of PS- $b$-P4VP and $0.25 \mathrm{mg}$ of $\mathrm{Zn}-2$ were dissolved in $1 \mathrm{~mL}$ of THF. Then, $4 \mathrm{~mL}$ of ultrapure water was added dropwise and THF was removed from the mixture by evaporation. In parallel, the inverse process, organicto-selective organic solvent, was also tested by preparing a solution of $3 \mathrm{mg}$ of PS- $b$-P4VP and $0.25 \mathrm{mg}$ of Zn-2 in THF, adding $4 \mathrm{~mL}$ of 1,4-dioxane dropwise and removing the THF by simple evaporation. Both experiments (organic-to-selective polar and organic-to-selective organic solvents) were also performed with the chiral homologue $\mathrm{Zn}$ - $(R, R, R, R)$-1-based metallocompound.

\section{RESULTS AND DISCUSSION}

With the aim to corroborate the effectiveness of the coordination of a $\mathrm{Zn}$-porphyrin with the pyridine units of the PS- $b$-P4VP (1:4), titration studies with the chiral metalloporphyrin $\mathrm{Zn}-(R, R, R, R)-\mathbf{1}$ and the achiral $\mathrm{Zn}-\mathbf{2}$ metalloporphyrin were conducted in solution using UV-vis absorption spectroscopy [35]. Zinc (II) porphyrins tend to bind with nitrogen axial ligands, resulting in a red-shift of the absorption bands due to an increase of the electron density of the porphyrin ring $[40,41]$. First, a titration study of the $\mathrm{Zn}-(R, R, R, R)-\mathbf{1}$ with pyridine to form $[\mathrm{Zn}-(R, R, R, R)-\mathbf{1}-(\mathrm{Py})]$ was followed by $\mathrm{UV}$-vis and used as a reference (Fig. S1). The titration was carried out at room temperature using a $5 \mu \mathrm{M}$ solution in dichloromethane of the metalloporphyrin (Sol. A) and consecutive additions of a mixed solution (Sol. B) of pyridine $(1.5 \mathrm{mM})$ and $\mathrm{Zn}-(R, R, R, R)-\mathbf{1}(5 \mu \mathrm{M})$ in dichloromethane. After the addition of $0.15 \mathrm{mM}$ of pyridine, a bathochromic shift of the Soret band from 423 $\mathrm{nm}$ to $430 \mathrm{~nm}$ was observed. The shift of the $\mathrm{Q}_{\alpha}$ band was from $550 \mathrm{~nm}$ to $564 \mathrm{~nm}$ and, for the lower energy $\mathrm{Q}$ band $\left(\mathrm{Q}_{\beta}\right)$, the shift was from $590 \mathrm{~nm}$ to $605 \mathrm{~nm}[40]$. Moreover, the $\mathrm{Q}_{\beta}$ band showed a clear increase in relative intensity with the increase of the pyridine amount. These results indicated the coordination of the pyridine with the Zinc (II) metal of the metalloporphyrin [41]. Towards the complexation BCP with the metalloporphyrins, a titration with the achiral $\mathrm{Zn}-2$ porphyrin was carried out at room temperature using a $50 \mu \mathrm{M}$ solution of the metalloporphyrin (Sol. A) in chloroform. Concurrently, in order to avoid any dilution factor of the porphyrin with respect to the $\mathrm{BCP}$ during the titration, a mixed solution composed by $47 \mu \mathrm{M}$ of $\mathrm{Zn}-2$ and $0.5 \mathrm{mM}$ of BCP in chloroform was prepared (Sol. B). The UV-vis absorption analysis started with the measurement of the absorption of the metalloporphyrin solution (Sol. A) 

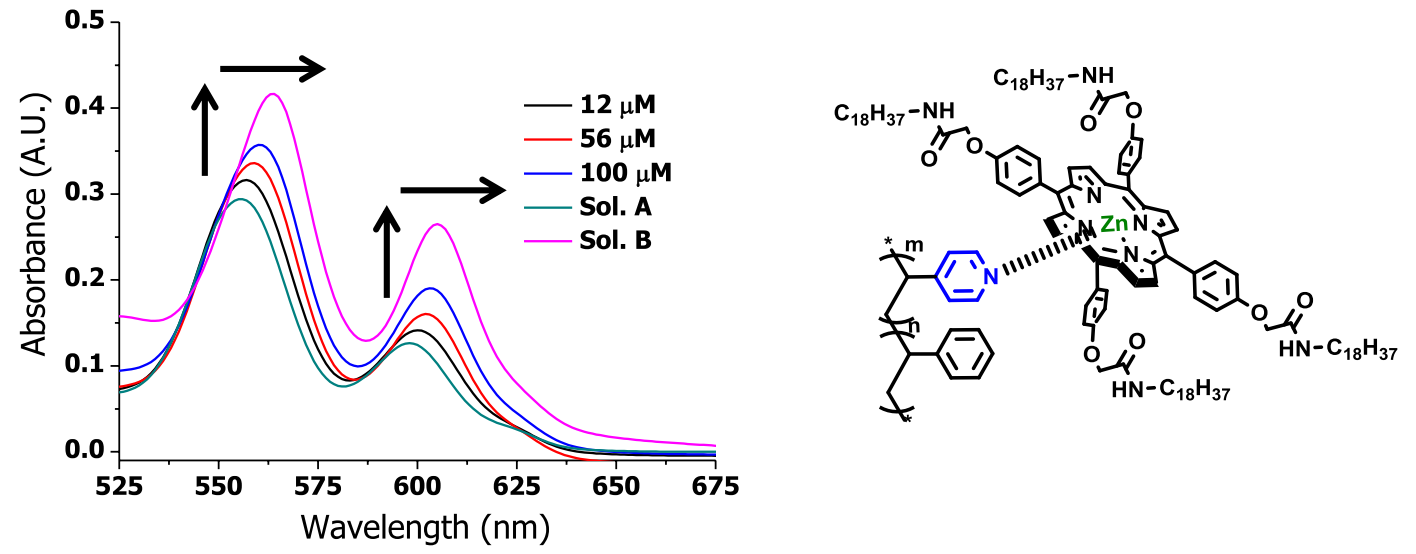

Fig. 2. UV-vis absorption spectra of the titration between Zn-2 (Sol. A) and Zn-2/PS-b-P4VP (Sol. B) to form the [Zn-2-(PS-b$\mathrm{P} 4 \mathrm{VP})]$ complex in chloroform

and continued with consecutive additions of Sol. B (Fig. 2). In addition to the coordination between the pyridine groups of the $\mathrm{BCP}$ and the zinc (II) metal ion in the core of the porphyrin, $\mathrm{Zn}-2$ can self-assemble by the coordination of the carbonyl group of the amide in the periphery with the zinc of an adjacent metalloporphyrin [37]. However, the non-coordinating solvent and conditions used in the titration studies avoid the self-aggregation of the metalloporphyrin to study exclusively the effects of the coordination among porphyrins and the BCP [36].

The UV-vis absorption spectra of the titration showed the progressive and characteristic evolution of the $\mathrm{Q}$ bands with the addition of BCP and the formation of the [Zn-2-(PS-b-P4VP)] complex (Fig. 2). Due to the concentrations used for the titration experiment, the signal of the Soret band was saturated and therefore, only the evolution of the $\mathrm{Q}$ bands was followed. The absorption band corresponding to the $\mathrm{Q}_{\alpha}$ band of the isolated $\mathrm{Zn}-\mathbf{2}$ metallocompound (Sol. A) at $556 \mathrm{~nm}$ increased in intensity and shifted to $564 \mathrm{~nm}$ with the increase of the of PS- $b$ P4VP concentration. Similarly, the $\mathrm{Q}_{\beta}$ band experienced a bathochromic shift from $598 \mathrm{~nm}$ to $605 \mathrm{~nm}$ when the binding to PS- $b$-P4VP occurred. The observed changes confirmed the axial coordination between the zinc (II) metal ion of the $\mathrm{Zn}-\mathbf{2}$ and the nitrogen of the pyridine in solution, and therefore the formation of the [Zn-2-(PS$b$-P4VP)] complex [35, 41]. The solution containing the complex was drop-cast on a quartz slide, and the film remaining after evaporation of the solvent was analyzed by UV-vis absorption spectroscopy. The spectrum of the film showed a similar bathochromic shift of the $\mathrm{Q}_{\alpha}$ and $\mathrm{Q}_{\beta}$ bands to the complex in solution (in both cases, $11 \mathrm{~nm}$ ) with respect to the pure Zn-2 solution (Fig. S2). These results showed the viability of casting the complex for the preparation of films of [Zn-2-(PS- $b$-P4VP)].

The formation of the complex between the chiral metalloporphyrin $\mathrm{Zn}-(R, R, R, R)-\mathbf{1}$ and the $\mathrm{BCP}$ was also proven both in solution and upon drop-casting onto quartz (Figs S3 and S4) by the red shift of the Q bands of the porphyrin. In solution, two isosbestic points appeared at $534 \mathrm{~nm}$ and at $588 \mathrm{~nm}$ in the UV-vis absorption spectra (Fig. S3). As a film, the complex showed a similar absorption spectrum to the achiral complex (Fig. S4).

Fluorescence spectroscopic measurements of the metalloporphyrins and their complexes also confirmed the formation of the supramolecular polymer. The spectra of the chiral metalloporphyrin and its complex with the BCP showed emission from the first excited state $\left(S_{1}\right)$ to the ground state $\left(S_{0}\right)$ upon excitation at $551 \mathrm{~nm}$ and $601 \mathrm{~nm}$ for metalloporphyrin $\mathrm{Zn}-(R, R, R, R)-\mathbf{1}$ and $554 \mathrm{~nm}$ and $601 \mathrm{~nm}$ for the complex [Zn- $(R, R, R, R)-1$-(PS- $b$ P4VP)], respectively (Fig. 3). The emission of the porphyrin in the complex was also red-shifted compared to the free compound. The $\mathrm{Q}_{\beta}$ band presented a larger Stokes shift $(62 \mathrm{~nm})$ for the [Zn- $(R, R, R, R)-\mathbf{1}-(\mathrm{PS}-b$ $\mathrm{P} 4 \mathrm{VP})]$ complex than for the isolated metalloporphyrin $\mathrm{Zn}-(R, R, R, R)-1(55 \mathrm{~nm})$, due to the electron-donating character of the pyridyl group $[42,43]$.

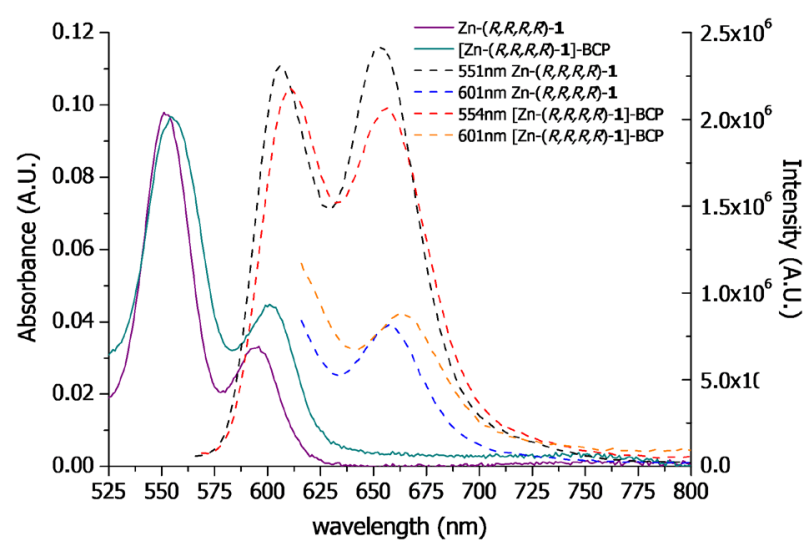

Fig. 3. UV-vis absorption and fluorescence spectra of $\mathrm{Zn}-(R, R, R, R)-1 \quad(5 \mu \mathrm{M})$ and [Zn- $(R, R, R, R)-\mathbf{1}-(\mathrm{PS}-b-\mathrm{P} 4 \mathrm{VP})]$ complex $(5 \mu \mathrm{M})$ in chloroform solution 


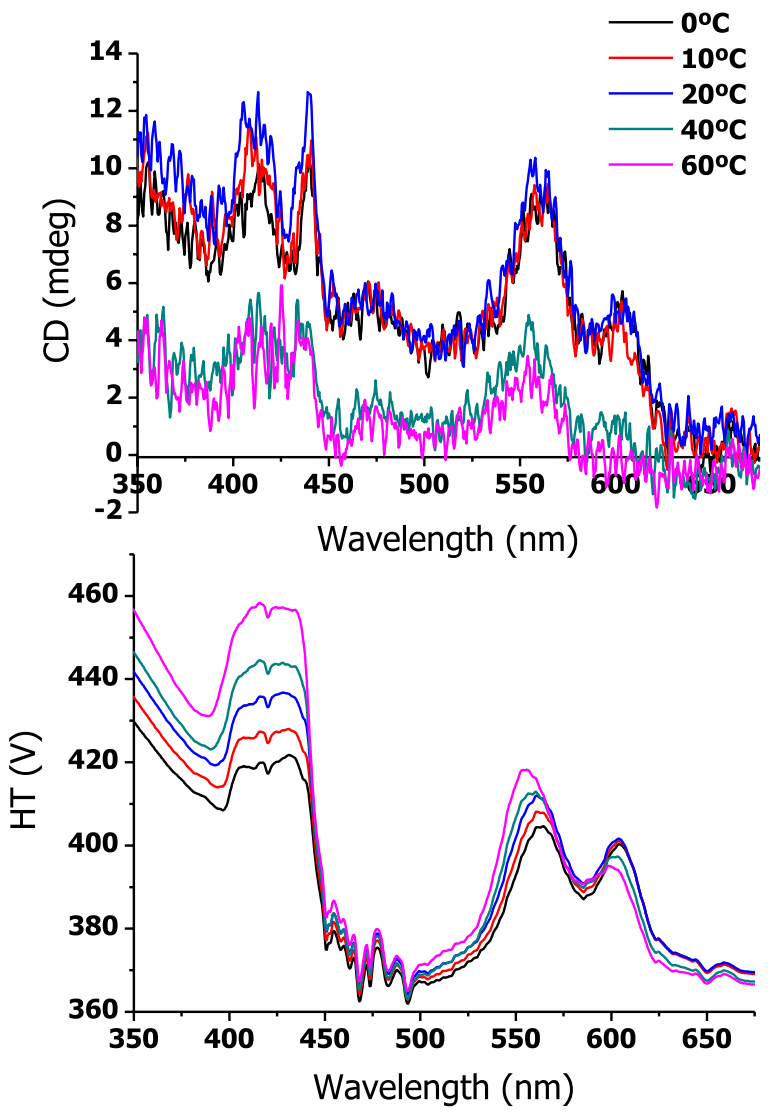

Fig. 4. $C D$ and corresponding and the corresponding absorption spectra of $[\mathrm{Zn}-(R, R, R, R)-\mathbf{1}-(\mathrm{PS}-b-\mathrm{P} 4 \mathrm{VP})]$ complex in chloroform solution $(50 \mu \mathrm{M})$ within the temperature range $0{ }^{\circ} \mathrm{C}$ to $60^{\circ} \mathrm{C}$. HT(V) means Hight Tension (Voltage)

The chirality present in the side-chains of $\mathrm{Zn}$ $(R, R, R, R)-\mathbf{1}$ could, in principle, lead to a chiral amplification, which could be observed through an increase of the optical activity of $\mathrm{Zn}-(R, R, R, R)-\mathbf{1}$-(PS- $b-\mathrm{P} 4 \mathrm{VP})]$ complex [12, 13, 44, 45]. Circular Dichroism (CD) spectroscopy is an extremely sensitivity probe for supramolecular structures [46]. Therefore, any proximity of porphyrin groups coordinated along the P4VP block should be detected using the technique. The complex was analyzed within a temperature range from $0^{\circ} \mathrm{C}$ to $60^{\circ} \mathrm{C}$ (Fig. 4). The CD spectra showed a slight temperature-dependence behavior of the $\mathrm{Zn}-(R, R, R, R)$-1-(PS- $b$ $\mathrm{P} 4 \mathrm{VP})]$ complex, being more evidence in the $\mathrm{Q}$ bands in the corresponding absorption spectra. From $0^{\circ} \mathrm{C}$ to $20^{\circ} \mathrm{C}$, clear CD signals were observed in the region of the Soret band and especially in the $\mathrm{Q}$ band area. The intensity of these signals was very low (a few millidegrees), which is characteristic of chromophores in a chiral environment that is dominated by the stereochemistry point rather than chiral order between chromophores (which is, generally, much more intense) [47]. With the increase of the temperature, a significant decrease in the optical activity, together with a slight blue shift of the Q-bands absorption were observed, suggesting a possible de-coordination of the pyridyl units of the $\mathrm{BCP}$ and the zinc (II) metal ion of the porphyrin. On the other hand, CD studies of the film of the $[\mathrm{Zn}-(R, R, R, R)-\mathbf{1}$-(PS- $b$-P4VP)] complex on quartz gave only a noisy and low intensity CD signal response (Fig. S5). This result indicated that the transmission of chirality from the stereogenic centers to the porphyrin organization was not effective in the complexes, where the average local achiral complex formation dominates. It should be pointed out that the pyridyl units can be occur locally chiral positions, although the atactic nature of the polymer meant that a regular interaction between porphyrins coordinated to the same chain was not possible.

${ }^{1} \mathrm{H}$ NMR experiments were also performed consecutively in solution to assess the binding between the metalloporphyrin and the BCP. Solutions of $\mathrm{Zn}-(R, R, R, R)-\mathbf{1}$, [Zn- $(R, R, R, R)-\mathbf{1}-(\mathrm{PS}-b-\mathrm{P} 4 \mathrm{VP})]$ complex and pure PS- $b$ $\mathrm{P} 4 \mathrm{VP}$ in deuterated chloroform were studied at $298 \mathrm{~K}$ (Fig. S6). Some differences between the [Zn- $(R, R, R, R)$ 1-(PS- $b$-P4VP)] complex and the PS- $b$-P4VP spectra were observed (Fig. S6). A slight shift and a broadening of the peaks positioned at 6.4 and $8.3 \mathrm{ppm}$ were experienced after the coordination. These peaks correspond to the protons of pyridine groups of the $\mathrm{BCP}$, whose changes were in concordance with the expected results $[35,48]$. Furthermore, the peak at $8.9 \mathrm{ppm}$ for $\mathrm{Zn}-(R, R, R, R)-\mathbf{1}$ shifted down to $8.7 \mathrm{ppm}$ when the porphyrin was coordinated, which corresponds to the protons of the pyrrole rings.

\section{Morphology of $[\mathrm{Zn}-2-(P S-b-P 4 V P)]$ and $[Z n-(R, R, R, R)-1-(P S-b-P 4 V P)]$ complexes}

The organization of the [Zn-2-(PS- $b$-P4VP)] and [Zn$(R, R, R, R)-1$-(PS- $b$-P4VP)] complexes at the microscopic level as materials was studied by Atomic Force Microscopy (AFM) (see Supporting information for sample preparation). The topography AFM images of [Zn-2-(PS$b$-P4VP)] (Figs 5a-5d) and [Zn- $(R, R, R, R)-\mathbf{1}-(\mathrm{PS}-b-$ $\mathrm{P} 4 \mathrm{VP})$ ] (Figs $5 \mathrm{e}-5 \mathrm{~h}$ ) showed apparently quite different morphology at larger length scales (several microns). The achiral complex depicted a quite flat surface with apparent very thin flake-like features and larger solid particlelike features protruding from the surface (Figs $5 \mathrm{a}-5 \mathrm{~d}$ ). On the other hand, the complex incorporating the chiral porphyrin evidenced a very regular morphology, where flake-like objects (about $0.5 \mu \mathrm{m}$ in width and 1-3 $\mu \mathrm{m}$ in length) were arranged randomly over the surface (Figs 5e-5h). Closer inspection of the surface (Fig. 5f) showed small round objects of approximately $20 \mathrm{~nm}$ high and $100 \mathrm{~nm}$ wide (Fig. 5h) localized between the flakes, which appeared rough but had no well-defined structure.

These features contrasted with those observed for the pure $\mathrm{Zn}-(R, R, R, R)-\mathbf{1}$, which arranged to form forming rounded dot-like features (average diameter of $0.5 \mathrm{~nm}$ ) (Fig. S7). This relevant difference in the packing highlighted the influence of incorporating the $\mathrm{BCP}$ in the selfassembly phenomenon of the complex. The morphology 

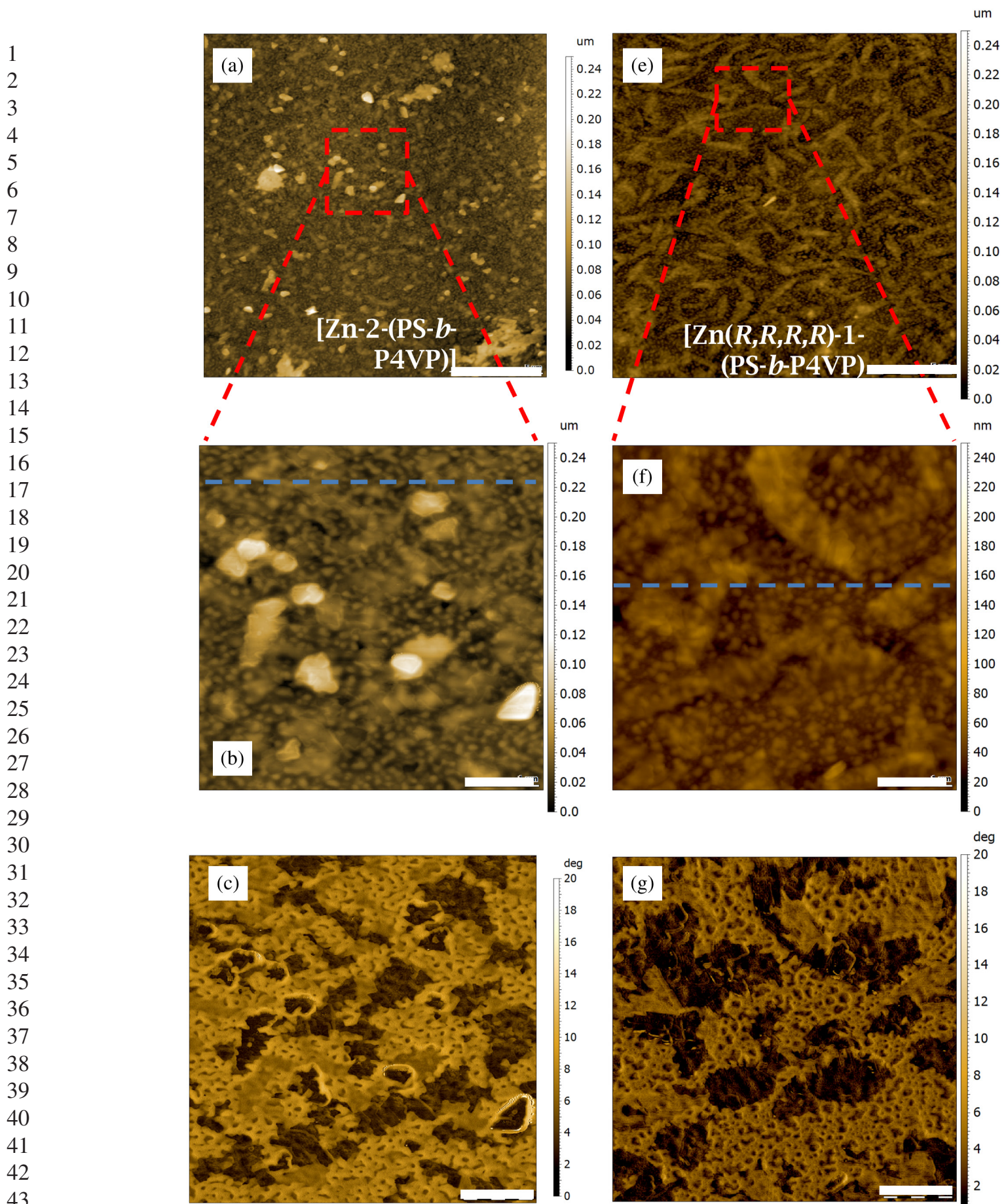

(d)
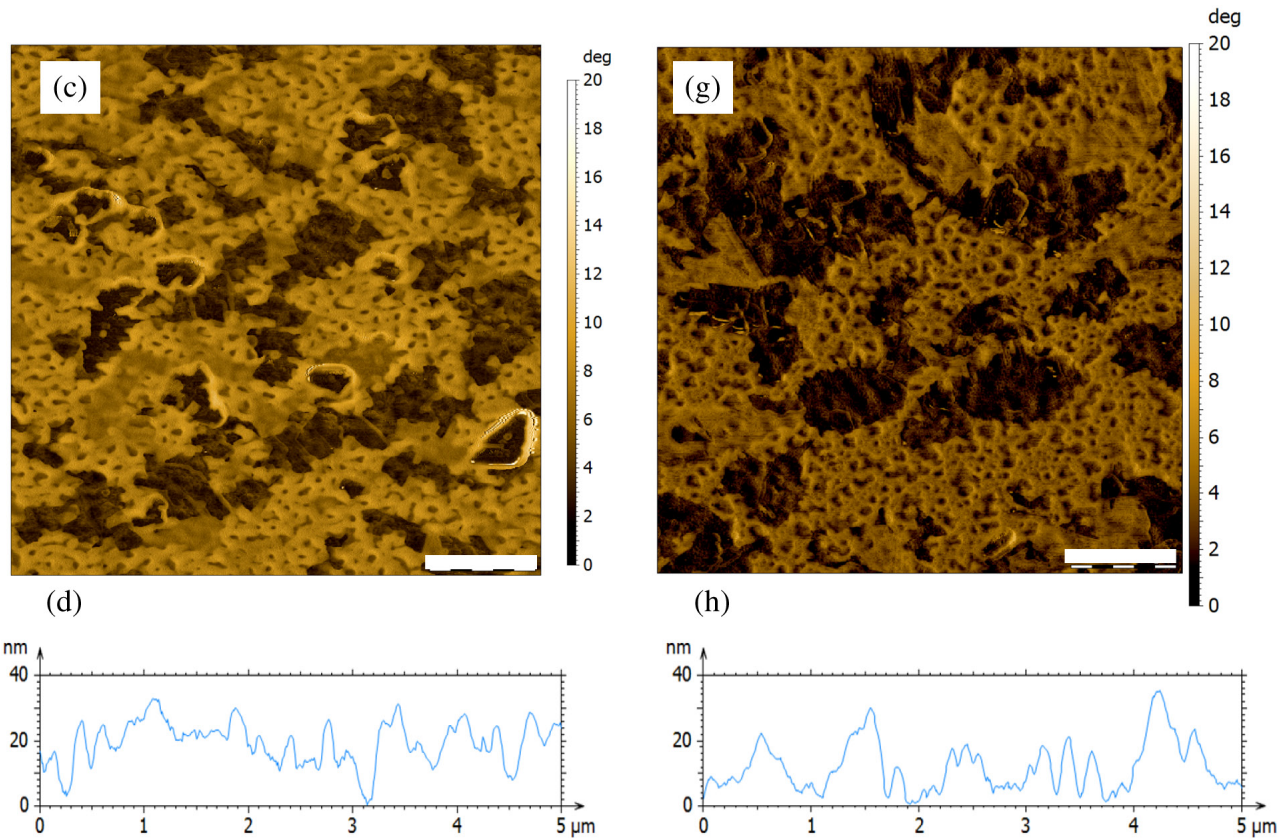

Fig. 5. AFM images of [Zn-2-(PS- $b$-P4VP) (a-d) and $[\mathrm{Zn}(R, R, R, R)-1-(\mathrm{PS}-b-\mathrm{P} 4 \mathrm{VP})](\mathrm{e}-\mathrm{h})$ coordinated compounds from a solution of chloroform drop-casted on HOPG surfaces. Topography images are shown in a and e (scale bars indicate $5 \mu \mathrm{m}$ ), b and f are closer-up topographic images from a and e (indicated with red dashed lines). Blue dashed lines indicate where the height profiles were taken from (scale bars indicate $1 \mu \mathrm{m}$ ), and $\mathrm{c}$ and $\mathrm{g}$ are phase images corresponding to topographic images b and $\mathrm{f}$ (scale bars 
of the achiral complex exhibited similar nanometer-scale round features with the previously mentioned larger particles overlaid (Fig. 5d). These larger particles, which appeared crystalline, were presumably the small molecules (since they were not present in the other complex) and were dark in the phase image from the AFM (Fig. 5c). In this phase image, the higher flake-like regions also appeared dark, indicating that they corresponded to the porphyrin component as well. In between these dark areas, quite well-defined nanostructured regions with dark dots were spotted, corresponding with the 20-nmtall dots, in both the achiral and chiral samples. Therecases, although it was more homogeneous in the case of the chiral porphyrin complex. These images contrasted with AFM images of pure PS- $b$-P4VP, prepared under same conditions (Fig. S8). The AFM topography of PS- $b$ P4VP (Fig. S8a) showed an irregular holed layer, probably as a consequence of the free evaporation of the solvent that created inhomogeneities during the evaporation, and consequently left locally dewetted regions. When the layer was analyzed closely, phase segregation from the continuous layer of the polymer was observed, although the holes were too large to obtain better contrasted fore, the nanostructure of the material was similar in both images (Fig. S8b). As a consequence of the large holes, not much information could be extracted from the phase image (Fig. S8c). In summary, both, height profiles of the achiral and chiral metalloporphyrin-based complexes exhibited flake-like structures of $\sim 35 \mathrm{~nm}$ in height and small spherical-shaped objects with averaged diameters of $\sim 50 \mathrm{~nm}$ and $\sim 100 \mathrm{~nm}$ in [Zn-2-(PS- $b$-P4VP)] and [Zn$(R, R, R, R)-1$-(PS- $b$-P4VP)], respectively (Figs $5 \mathrm{~d}$ and $5 \mathrm{~h}$ ). Contrarily, the size of the objects observed in the pure polymer was $\sim 10 \mathrm{~nm}$ (Fig. S8d).

Transmission Electron Microscopy (TEM) was used to determine the influence of the chirality of the metalloporphyrins in the phase segregation of the complexes. Separate solutions of PS- $b$-P4VP, [Zn-2-(PS- $b$-P4VP)] and $[\mathrm{Zn}-(R, R, R, R)-1-(\mathrm{PS}-b-\mathrm{P} 4 \mathrm{VP})]$ in chloroform were dried under vacuum for several hours until solid powders were afforded. The final powders were separately embedded in an epoxy resin and cured at $60^{\circ} \mathrm{C}$ for 2 days. Then, they were cut by using a microtome to finally obtain solid films with a thickness of 70-100 nm. The different samples were collected on holey-carbon copper TEM grids and were also selectively stained with iodine vapors for $1 \mathrm{~h}$ in a sealed container. TEM images of the pure block copolymer showed phase segregation with
Fig. 6. TEM images of (a) the pure PS- $b$-P4VP, and the porphyrin complexes (b) [Zn-2-(PS- $b$-P4VP)] and (c) [Zn-( R,R,R,R)-1-(PS- $\quad 56$ $b$-P4VP)], obtained by microtoming solid samples from a dried chloroform solution (Scale bars indicate $100 \mathrm{~nm}$ ) 

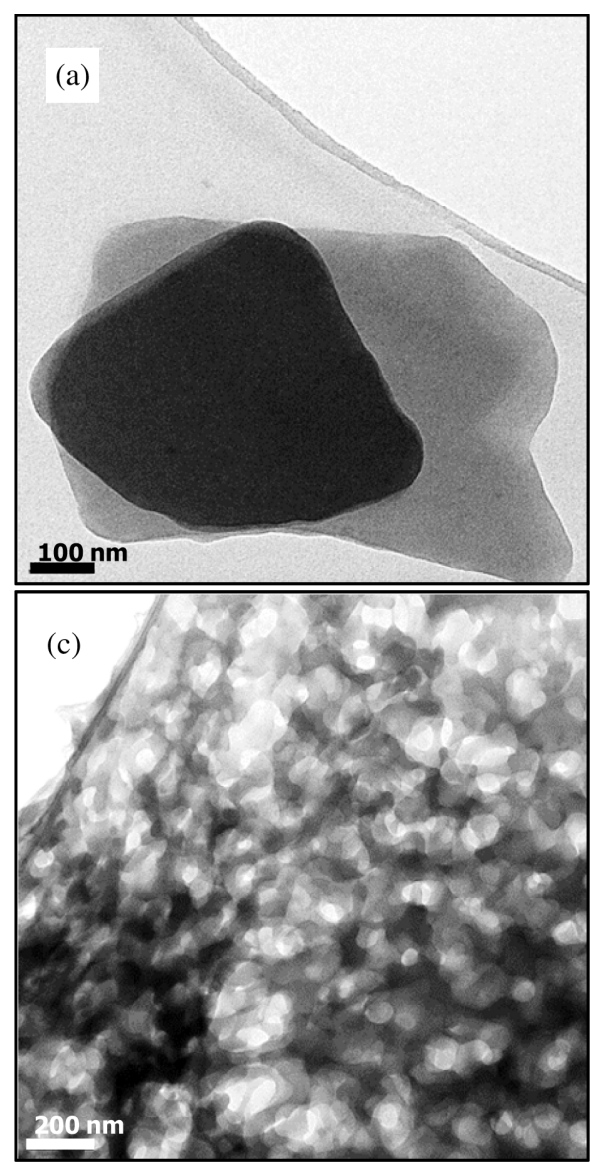

Fig. 7. TEM images of Zn-porphyrins and their complexes with PS- $b$-P4VP obtained from solutions in ethanol by drop-casting on carbon coated copper (a) Zn-2 (scale bar indicates $100 \mathrm{~nm}$ ), (b) Zn- $(R, R, R, R)-\mathbf{1}$ (scale bar indicates $100 \mathrm{~nm})$, (c) [Zn-2-(PS- $b$ $\mathrm{P} 4 \mathrm{VP})]$ (scale bar indicates $200 \mathrm{~nm}),(\mathrm{d})[\mathrm{Zn}-(R, R, R, R)-\mathbf{1}-(\mathrm{PS}-b-\mathrm{P} 4 \mathrm{VP})]$ (scale bar indicates $50 \mathrm{~nm})$ a quasi-lamellar structure with darker domains corresponding to the P4VP block (as a consequence of the staining used to enhance TEM contrast) (Fig. 6a). The [Zn-2-(PS- $b$-P4VP)] complex showed an unclear order (Fig. 6b). However, [Zn-(R,R,R,R)-1-(PS- $b-\mathrm{P} 4 \mathrm{VP})]$ chiral complex displayed very defined domains, dark areas with bright spots, probably arranging either in body-centered cubic spheres (fm3m), gyroid (ia3d) or inverse discontinuous cubic structure (fd3m) (Fig. 6c). This evidence proved the effect of the presence of stereogenic centers in the alkyl chains of the porphyrin chains on the self-assembly of the supramolecular PS$b$-P4VP complex, resulting in segregated regions with different structures. The chiral characteristic, given only by a single methyl group in each substituted alkyl chain, enlarged significantly the space domains between PS and $\mathrm{P} 4 \mathrm{VP}$, probably because of the higher steric hindrance between neighboring metalloporphyrin units anchored to the pyridyl groups.

While the coordination of the porphyrin to the $\mathrm{BCP}$ in the composite material is clear, an alternative assembly strategy to obtain nanostructured materials was studied by modifying the polarity of solvents to obtain polymeric doped micelles [49-51]. Li and coworkers reported chiral

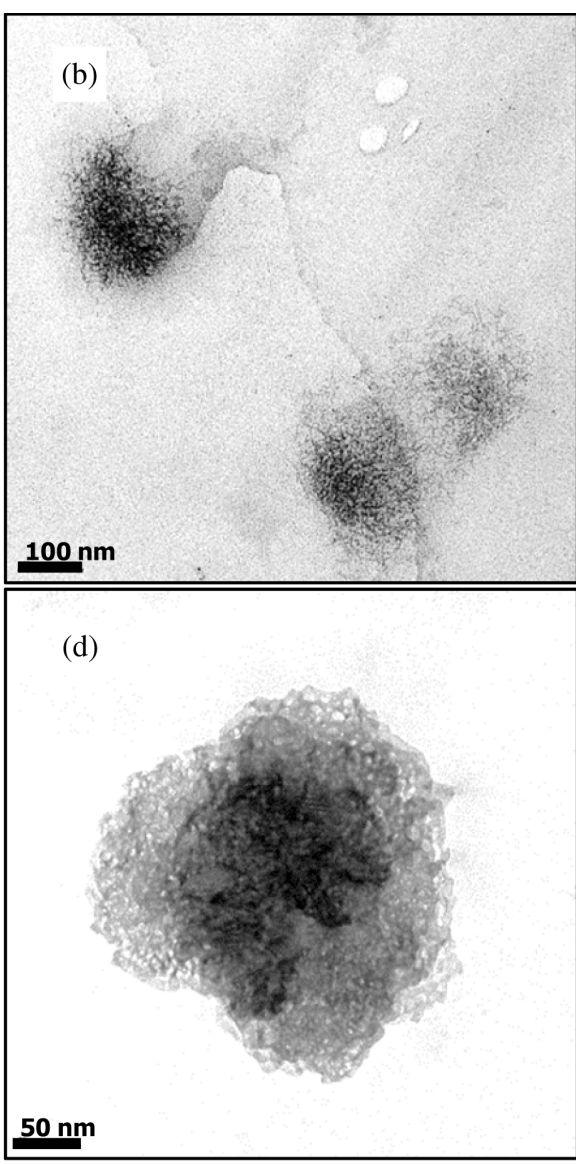



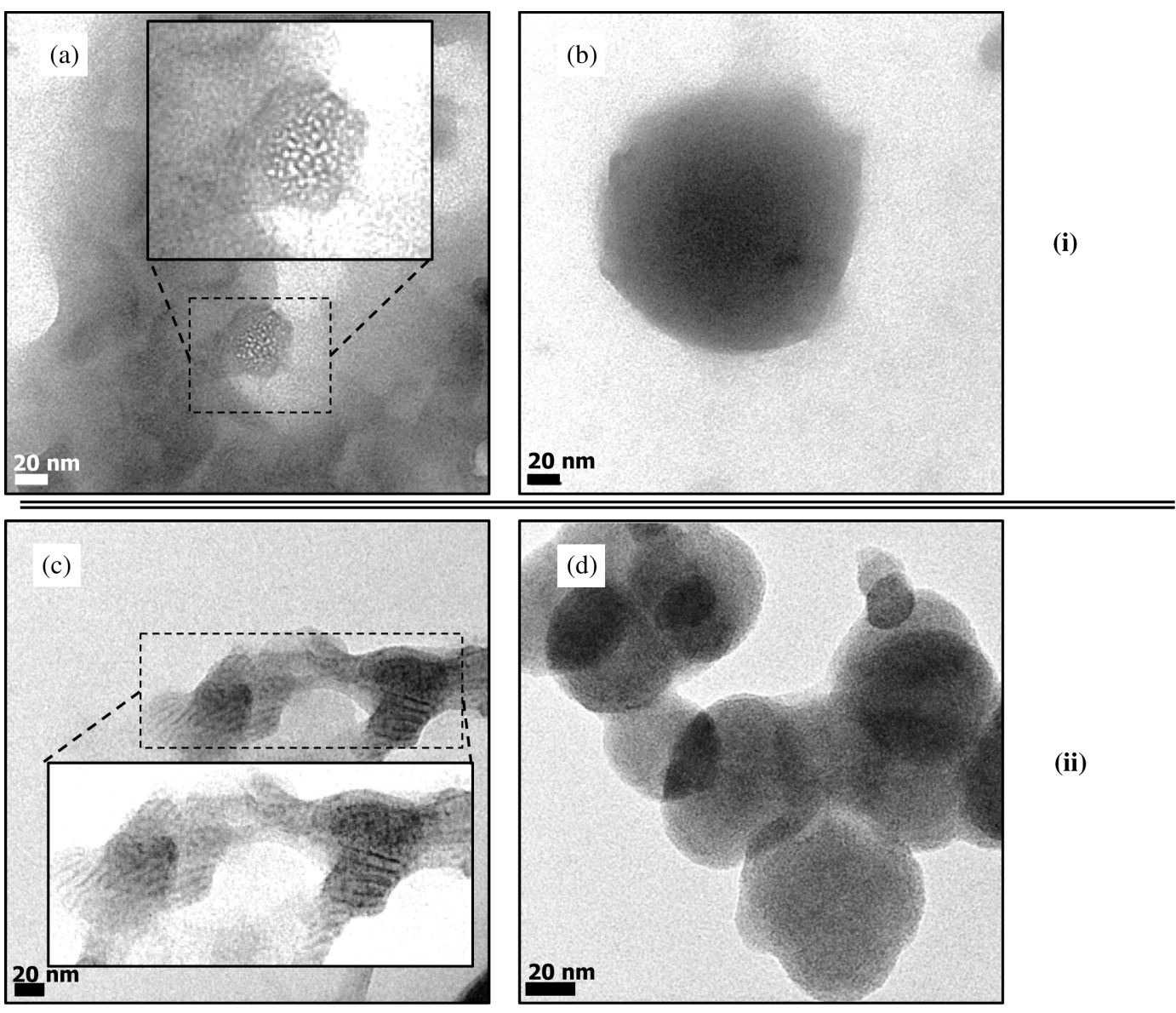

(ii)

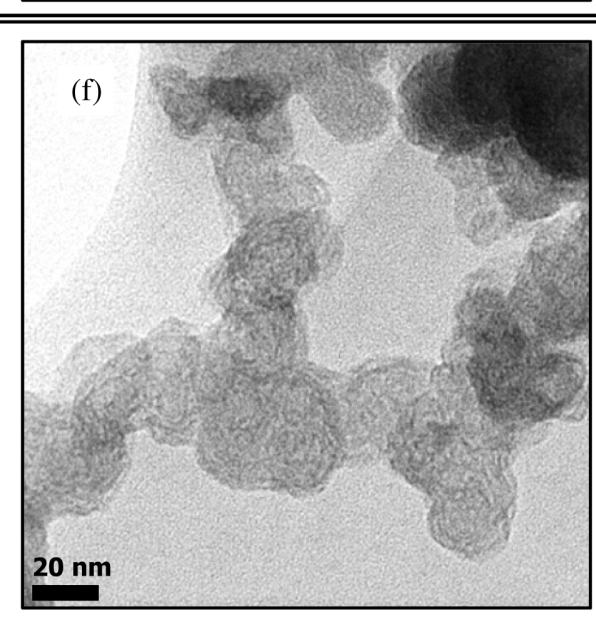

Fig. 8. TEM images of $[\mathrm{Zn}-(R, R, R, R)-\mathbf{1}$-(PS- $b$-P4VP) $]$ samples prepared from ethanol. (a) and (b) from a drop of the mixed solution without staining, (c) and (d) from the dried mixed solution embedded in an epoxy resin without staining and, (e) and (f) from dried mixed solution embedded in an epoxy resin stained with $\mathrm{I}_{2}$ vapors (Scale bars indicate $20 \mathrm{~nm}$ )

than in $\mathrm{Zn}-\mathbf{2}$. In the $[\mathrm{Zn}-(R, R, R, R)-\mathbf{1}-(\mathrm{PS}-b-\mathrm{P} 4 \mathrm{VP})]$ complex (Fig. 7d), well-defined "cauliflower"-shaped structures were observed. A significantly more fibrous structure at the corona of the "flowers" suggested that polymer coiled with $\mathrm{Zn}-(R, R, R, R)-\mathbf{1}$, leading to twisted fibrous meshes.

To further study the [Zn- $(R, R, R, R)-\mathbf{1}-(\mathrm{PS}-b$-P4VP)] complex morphology in ethanol, different preparation methodologies of the TEM samples were tested: (i) a drop of solution was cast on a holey-carbon copper TEM grid and directly analyzed, (ii) an aliquot of the solution was dried and then was embedded in an epoxy resin, the resin was cured and then, cut using microtome in thin slices $(70-100 \mathrm{~nm})$ and analyzed by TEM without staining, and (iii) the same procedure as (ii) but the sample was stained with $\mathrm{I}_{2}$ vapors for $1 \mathrm{~h}$ in a sealed container before TEM imaging (Fig. 8). All the samples indicated similar superstructures, albeit with varying degrees of detail (Fig. 8). 
1 From the drop of the solution of the $\mathrm{Zn}-(R, R, R, R)$ 2 1-(PS- $b$-P4VP)] complex analyzed directly by TEM 3 (Figs 8a-8b), some regions with agglomerated matter and 4 spherical aggregates with blurred perimeter were appre5 ciated. The majority of the round objects were small, 6 but within a broad range of sizes (from 40 to $300 \mathrm{~nm}$ ). 7 The more continuous film seen in Fig. 8a indicated a 8 twisted-layer type structure. When the sample was pre9 pared with epoxy resin, self-assembled $\mathrm{Zn}-(R, R, R, R)-\mathbf{1}$ porphyrin domains became clearly visible in the continuous-type structure (Fig. 8c), and the aggregated particles were spotted, although no internal structure was evident (Fig. 8d). The dark regions in Fig. 8c probably corresponded to regions containing $\mathrm{Zn}-(R, R, R, R)-\mathbf{1}$ porphyrin, forming stripes of $4 \mathrm{~nm}$ of separation, because the sample was not selectively stained. After enhancing the contrast by selectively staining the P4VP domains with $I_{2}$ vapors, the distribution of the $\mathrm{BCP}$ in the $[\mathrm{Zn}-(R, R, R, R)-\mathbf{1}-(\mathrm{PS}-b$ $\mathrm{P} 4 \mathrm{VP})]$ micelles could be clearly appreciated as layers of $\sim 4 \mathrm{~nm}$ of material (Figs 8e-8f). Images illustrated a possible twisted rope-like structure which could belong to P4VP domains, possibly coiled by the chiral effect of the porphyrin, although no handedness could be determined from the images.

With the aim to elucidate if the self-assembled complex in the corona of micelles in ethanol, corresponding to the $[\mathrm{Zn}-(R, R, R, R)-\mathbf{1}-(\mathrm{PS}-b-\mathrm{P} 4 \mathrm{VP})]$ complex, was optically active, $\mathrm{CD}$ measurements of the micelles in solution were performed (Fig. S9). However, no indication of chiral packing was observed, suggesting that no chiral chromophore interaction was present in the materials, and consequently, no significant chiral superstructure could be evidenced.

An alternative way to form the complex between the porphyrin and the BCP is by the solvent exchange methodology [54, 55]. The solvent exchange procedure involves, first, dissolving the amphiphilic copolymer in a non-selective solvent and, secondly, slowly adding a selective solvent until the latter is in excess. Then the nonselective solvent is removed (usually by evaporation), leading to a solvent exchange. In principle, progressive evaporation of the non-selective component induced the collapse of the solvophobic part of the copolymer, leading to a micelle-like structure (Scheme 1). To prove this, organic-to-selective polar solvent exchange was tested in the present systems.

PS- $b$-P4VP and the corresponding $\mathrm{Zn}-2$ or $\mathrm{Zn}$ $(R, R, R, R)-1$ porphyrin were dissolved in THF, ultrapure water was added dropwise and THF was removed from the mixture by evaporation. In parallel, the inverse process, organic-to-selective organic solvent, was also tested by the preparation of a solution of PS- $b$ $\mathrm{P} 4 \mathrm{VP}$ and $\mathrm{Zn-2}$ or $\mathrm{Zn}-(R, R, R, R)-\mathbf{1}$ in THF, addition of 1,4-dioxane dropwise and removal of the THF by simple evaporation. Additionally, control experiments for each solvent exchange process with pure PS- $b$-P4VP, $\mathrm{Zn-2}$ and $\mathrm{Zn}-(R, R, R, R)-\mathbf{1}$ under the same conditions
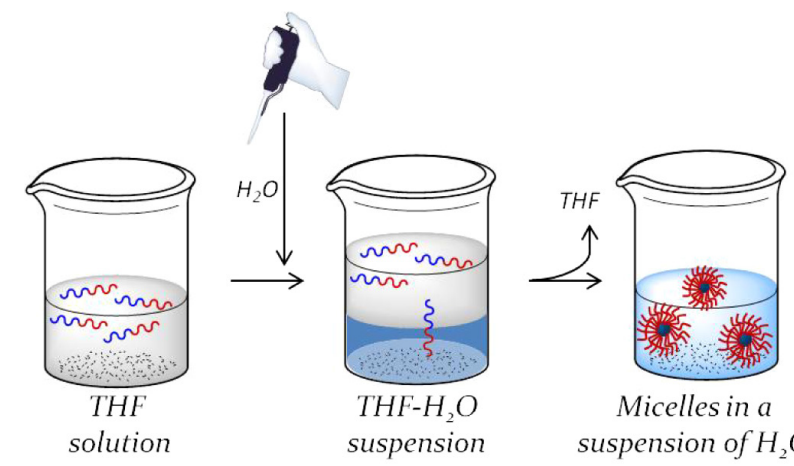

Micelles in a suspension of $\mathrm{H}_{2} \mathrm{O}$

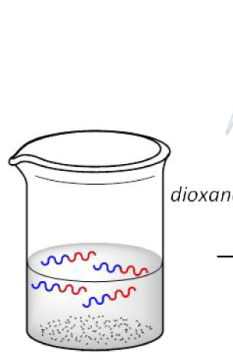

THF

solution
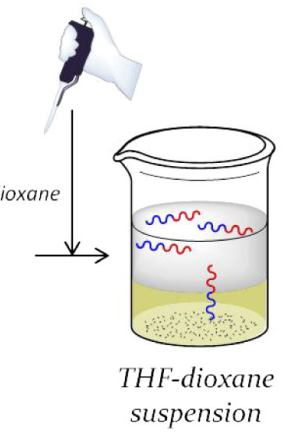

$\sim=\mathrm{PS} \frown=\mathrm{P}_{4} \mathrm{VP}$

Scheme 1. Representation of the formation of the PS- $b-\mathrm{P} 4 \mathrm{VP}$ micelles depending on the polarity of the solvent (solvent exchange method)

and following the same procedures were performed. A drop of each final mixture was separately analyzed by TEM using holey-carbon copper grids. TEM images of PS- $b$-P4VP in water (Fig. S10) revealed well-defined round micelle-like objects with a narrow range of sizes $(\sim 150-400 \mathrm{~nm})$. The P4VP phase was selectively stained with iodine vapors, appearing as dark regions. Klinger et al. observed similar micelles of PS- $b$-P2VP following a similar procedure [56]. When the solvent inducing the micellar assembly was 1,4-dioxane, a core of P4VP surrounded by a PS corona was expected [57]. TEM images of this sample showed smaller micellar structures than in water which were surrounded by poorly defined matter (blurry appearance) (Fig. S11). After staining, the P4VP domains appeared spherical (darker regions in the images) surrounded by the PS block, probably swollen as a result of the contact with 1,4-dioxane, and consequently, forming blurred matter in their surroundings [58]. The volume fraction of the blocks in the PS- $b$-P4VP used here (molar ratio PS:P4VP of 1:4) surely determined sizes, distributions and behavior of micelle-like objects in the different solvents studied, due to the selectivity of each block. The TEM image of pure Zn-2 in water showed straight, planar and large ribbon morphologies (Fig. S11a) [59]. However, when 1,4-dioxane was used, $\mathrm{Zn}-2$ precipitated (Fig. S11b), forming mixed structures, plates (as 


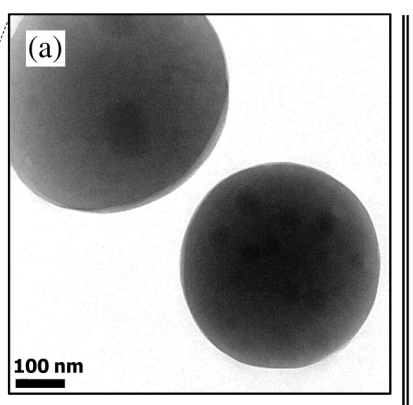

(b) water

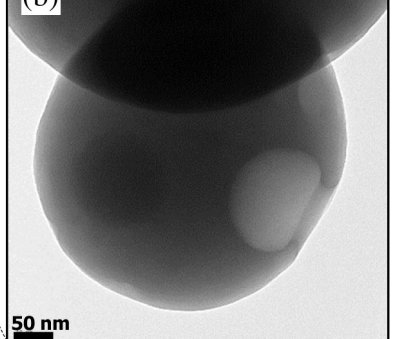

$50 \mathrm{~nm}$

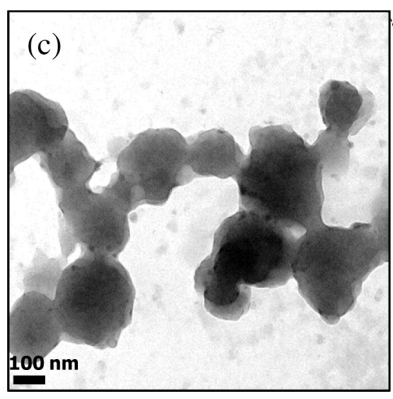

(d)

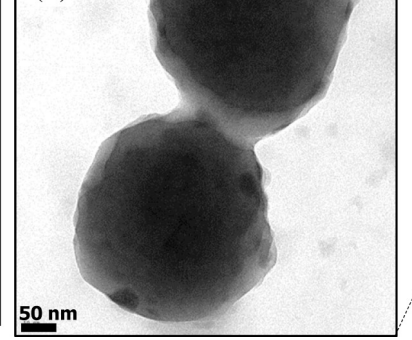

dioxane

Fig. 9. Pictures of [Zn-2-(PS- $b$-P4VP)] solutions in water (greenish) and in 1,4-dioxane (pale rose). TEM images of [Zn-2-(PS- $b$ $\mathrm{P} 4 \mathrm{VP}$ )] micelles in water (a) and (b) and in 1,4-dioxane (c) and (d) (a and c scale bars indicate $100 \mathrm{~nm}$; b and d scale bars indicate $50 \mathrm{~nm}$ )
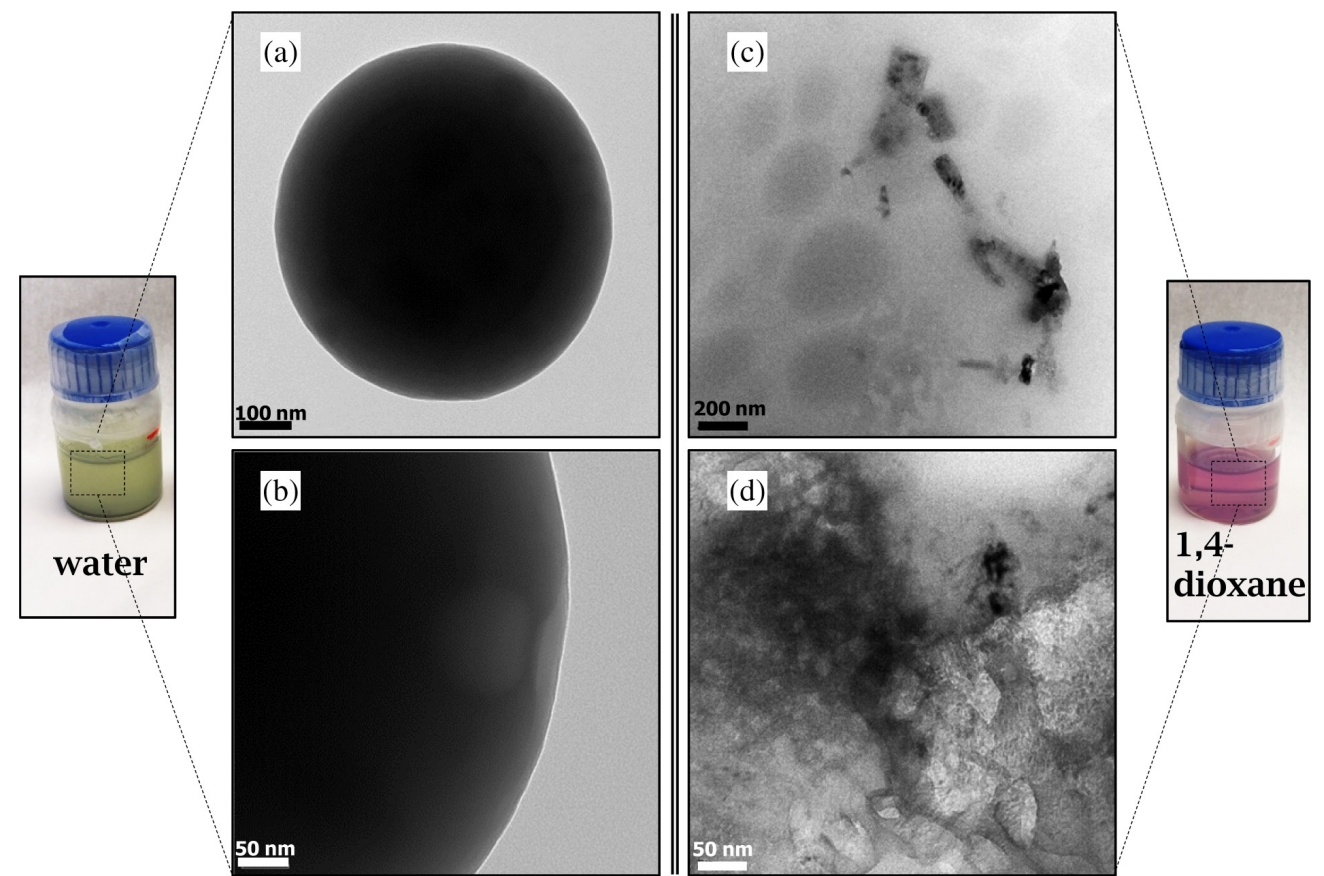

Fig. 10. Pictures of $[\mathrm{Zn}-(R, R, R, R)-1-(\mathrm{PS}-b-\mathrm{P} 4 \mathrm{VP})]$ solutions in water (greenish) and 1,4-dioxane (pink) and TEM images of: (a) and (b) $[\mathrm{Zn}-(R, R, R, R)-\mathbf{1}-(\mathrm{PS}-b$-P4VP)] micelles in water and, (c) and (d) [Zn- $(R, R, R, R)-1$-(PS- $b$-P4VP)] mixed compound in 1,4-dioxane (a scale bar indicates $100 \mathrm{~nm}$; b scale bar indicates $50 \mathrm{~nm}$; c scale bar indicates $200 \mathrm{~nm}$; d scale bar indicates $50 \mathrm{~nm}$ )

observed from ethanol) as well as wider ribbons due to the coordinative character of the solvent [60]. Moreover, TEM images of the chiral $\mathrm{Zn}-(R, R, R, R)-\mathbf{1}$ precipitated in water (Fig. S11c) depicted very small round aggregates (sub-20 nm) with barely discernible fibrous substructures, very different from the achiral homologue.
When the solvent was 1,4-dioxane (Fig. S11d), similar aggregates to those in water were found, although with a larger surface (approximately $80 \mathrm{~nm}$ ) with internal fibrous nanostructures.

On the other hand, TEM images of [Zn-2-(PS- $b$ P4VP)] formed very well-defined round and filled 
spheres (Figs 9a-9d). While in water and after THF evaporation, the spheres had a diameter of approximately $500 \mathrm{~nm}$ (Figs 9a-9b); from the 1,4-dioxane mixture, the spheres had diameters between 200 and $300 \mathrm{~nm}$, with a rougher appearance (Figs 9c-9d). In both samples, darker spots were also seen inside the spheres, probably due to enriched porphyrin localized areas. For the sample prepared in water, in which the hydrophilic pyridyl fragments seems to be in contact with the water, unlike the organic solvent exchange, rougher and smaller particles with clear coronas (presumably composed of PS domains) were observed (Figs 9a-9b). An increased tendency towards aggregation between the particles in 1,4-dioxane was appreciated, due to the affinity between alkyl chains of neighboring packed porphyrins that could be swelled by the organic solvent (Figs 9c-9d).

The chiral complex [Zn- $(R, R, R, R)-\mathbf{1}-(\mathrm{PS}-b-\mathrm{P} 4 \mathrm{VP})]$ also formed well-defined particles in water, approximately $500 \mathrm{~nm}$ in diameter (Figs 10a-10b), similar to its achiral homologue. In contrast, when the chiral metallocompound was immersed in 1,4-dioxane (Figs 10c-10d), no particulate aggregation was observed, and only polymer phase segregation and aggregates could be discerned (Fig. 10c). Apparently, no coordination between the PS$b$-P4VP and Zn- $(R, R, R, R)-\mathbf{1}$ was concluded, and coordination of the zinc (II) with the 1,4-dioxane occurred [60]. This fact was assumed because of the higher solubility of the chiral porphyrin in contrast to the achiral one, and the large excess of solvent used compared to the polymer amount. Snitka et al. observed similar striped structures (they designated them as molecular ribbons) in aggregates of tetrakis(4-sulfonatophenyl)porphyrin, supporting the coordination of the precursors by the solvent [61].

\section{CONCLUSIONS}

The binding of both achiral and chiral zinc (II) porphyrins to the pyridyl block of the polymer is clear both in solution in a non-coordinating solvent (chloroform) and in films. The system can also be used to make nanomaterials whose morphology depends on the chiral or non-chiral form of the coordinated zinc (II) porphyrin analogue. The coordinated systems [Zn-2-(PS- $b$-P4VP)] and $[\mathrm{Zn}-(R, R, R, R)-1-(\mathrm{PS}-b$-P4VP)] have differences in their morphology as cast materials, observing more uniformity for the chiral complex as well as when precipitation under far-from-equilibrium conditions occurred. Notably, the precipitation in water generates the most homogeneous material although no well-defined substructure exists in the particle generated. The chiral complex does not show significant optical activity either in solution or in the nanomaterials prepared, indicating that the polymer coordination prevents the proximity of the chromophores and stereogenic centers. This situation contrasts dramatically from the situation in which the pure porphyrins stack on top of one another thanks to hydrogen bonding, leading to significant optical activity. It also indicates that this kind of polymer with an atactic and rigid structure in combination with the necessary axial coordination of the pyridine groups and the chiral zinc (II) porphyrins is not suited. Therefore, a block copolymer with a control of the tacticity and phase segregation will be more appropriate for further studies of chirality transfer in this kind of dye-polymer hybrid material.

\section{Acknowledgements}

We thank T. Parella (UAB) for help in DOSY-NMR measurements, J. Oró (ICMAB) for help in TEM imaging and M. Simón (ICMAB) for help in AFM imaging. The work was supported by MINECO with the Project MAT2016-77852-C2-1-R (AEI/FEDER, UE) and MAT2013-47869-C4-2-P and Generalitat de Catalunya for the project 2017-SGR-1277. AGC acknowledges financial support from the Spanish Ministry of Economy and Competitiveness through the "Severo Ochoa" Programme for Centres of Excellence in R\&D (SEV- 2015-0496) and the Ministry of Science, Innovation and Universities for a Ramon y Cajal contract (RYC-2017-22910).

\section{Supporting information}

Additional information on coordination studies and synthesis and characterization studies, including Figs. S1S11 are available are given in the supplementary material. This material is available free of charge via the Internet at http://www.worldscinet.com/jpp/jpp.shtml.

\section{REFERENCES}

1. Harada A. Supramolecular Polymer Chemistry, Wiley-VCH Verlag GmbH \& Co. KGaA: Weinheim, Germany, 2011.

2. Thibault RJ and Rotello VM. In Molecular Recognition and Polymers, John Wiley \& Sons, Inc., Hoboken, NJ, 2008; 1-7.

3. Elemans JAAW, VanHameren R, Nolte RJM and Rowan AE. Adv. Mater. 2006; 18: 1251-1266.

4. Yan Q, Luo Z, Cai K, Ma Y and Zhao D. Chem. Soc. Rev. 2014; 43: 4199-4221.

5. Zhang C, Chen P, Dong H, Zhen Y, Liu M and Hu W. Adv. Mater. 2015; 27: 5379-5387.

6. Aragonès AC, Darwish N, Saletra WJ, Pérez-García L, Sanz F, Puigmartí-Luis J, Amabilino DB and Díez-Pérez I. Nano Lett. 2014; 14: 4751-4756.

7. Imahori H. J. Phys. Chem. B. 2004; 108: 6130-6143.

8. Louahem M'Sabah B, Boucharef M, Warnan J, Pellegrin Y, Blart E, Lucas B, Odobel F and Boucle J. Phys. Chem. Chem. Phys. 2015; 17: 9910-9918.

9. Goldberg I. Chem. Comm. 2005; 10: 1243-1254.

10. Chmielewski PJ and Latos-Grażyński L. Coord. Chem. Rev. 2005; 249: 2510-2533. 
11. Pasternack RF, Giannetto A, Pagano P and Gibbs EJ. J. Am. Chem. Soc. 1991; 113: 7799-7800.

12. Beletskaya I, Tyurin VS, Tsivadze AY, Guilard R and Stern C. Chem. Rev. 2009; 109: 1659-1713.

13. Ogi S, Ikeda T, Wakabayashi R, Shinkai S and Takeuchi M. Chem. -Eur. J. 2010; 16: 8285-8290.

14. Sguerra F, Bulach V and Hosseini MW. Dalton Trans. 2012; 41: 14683-14689.

15. Puigmarti-Luis J, Saletra WJ, Gonzalez A, Amabilino DB and Pérez-García L. Chem. Comm. 2014; 50: $82-84$.

16. Hamley IW. Prog. Polym. Sci. 2009; 34: 1161-1210.

17. Kim JK, Yang SY, Lee Y and Kim Y. Prog. Polym. Sci. 2010; 35: 1325-1349.

18. Heier J, Kramer EJ, Walheim S and Krausch G. Macromolecules 1997; 30: 6610-6614.

19. Albert JNL and Epps III TH. Mater. Today 2010; 13: 24-33.

20. Bates FS and Fredrickson GH. Phys. Today 1999; 52: 32-38.

21. Gowd EB, Böhme M, Stamm M. IOP C. Ser. Mater. Sci. Eng. 2010; 14: 012015.

22. Sinturel C, Vayer M, Morris M and Hillmyer MA. Macromolecules 2013; 46: 5399-5415.

23. Kao J, Thorkelsson K, Bai P, Rancatore BJ and Xu T. Chem. Soc. Rev. 2013; 42: 2654-2678.

24. Gowd EB, Koga T, Endoh MK, Kumar KV and Stamm M. Soft Matter 2014; 10: 7753-7761.

25. D'Aprano A and Fuoss RM. J. Polym. Sci. 1969; 7: 1101-1109.

26. Letchford K and Burt H. Eur. J. Pharm. Biopharm. 2007; 65: 259-269.

27. Li X and Han Y. J. Mater. Chem. 2011; 21: 18024-18033.

28. Radjabian M, Koll J, Buhr K, Handge UA and Abetz V. Polymer 2013; 54: 1803-1812.

29. Clodt JI, Filiz V, Rangou S, Buhr K, Abetz C, Höche D, Hahn J, Jung A and Abetz V. Adv. Funct. Mater. 2013; 23: 731-738.

30. Klinger D, Wang CX, Connal LA, Audus DJ, Jang SG, Kraemer S, Killops KL, Fredrickson GH, Kramer EJ and Hawker CJ. Angew. Chem., Int. Ed. 2014; 53: 7018-7022.

31. Bronstein LM, Sidorov SN, Valetsky PM, Hartmann J, Cölfen H and Antonietti M. Langmuir 1999; 15: 6256-6262.

32. Guldi DM, Rahman GM, Qin S, Tchoul M, Ford WT, Marcaccio M, Paolucci D, Paolucci F, Campidelli S and Prato M. Chem. - Eur. J. 2006; 12: 2152-2161.

33. Arulkashmir A, Mahale RY, Dharmapurikar SS, Jangid MK and Krishnamoorthy K. Polym. Chem. 2012; 3: 1641-1646.

34. Huang HX, Liu J and Cai YQ. J. Lumin. 2013; 143: 447-452.

35. Gao B, Wang R and Du R. J. Porphyrins Phthalocyanines 2010; 14: 235-243.
36. Oliveras-González C, Di Meo F, González-Campo A, Beljonne D, Norman P, Simón-Sorbed M, Linares M and Amabilino DB. J. Am. Chem. Soc. 2015; 137: 15795-15808.

37. Feldborg LN, Saletra WJ, Iavicoli P and Amabilino DB. J. Porphyrins Phthalocyanines 2011; 15: 995-1003.

38. Iavicoli $\mathrm{P}, \mathrm{Xu} \mathrm{H}$, Feldborg LN, Linares M, Paradinas M, Stafström S, Ocal C, Nieto-Ortega B, Casado J, López Navarrete JT, Lazzaroni R, Feyter SD and Amabilino DB. J. Am. Chem. Soc. 2010; 132: 9350-9362.

39. Varshney SK, Zhong XF and Eisenberg A. Macromolecules 1993; 26: 701-706.

40. Gautam R and Chauhan SMS. Mater. Sci. Eng. C Mater. 2014; 43: 447-457.

41. Alessio E. Structure and Bonds: Non-Covalent Multi-Porphyrin Assemblies: Synthesis and Properties, Springer, 2006; Vol. 121, pp. 1-47.

42. Apanasovich VV, Novikov EG, Yatskov NN, Koehorst RBM, Schaafsma TJ and van Hoek A. J. Appl. Spectrosc. 1999; 66: 613-616.

43. Karolczak J. Kowalska D, Lukaszewicz A, Maciejewski A and Steer RP. J. Phys. Chem. A 2004; 108: 4570-4575.

44. Green MM, Reidy MP, Johnson RD, Darling G, O'Leary DJ and Willson G. J. Am. Chem. Soc. 1989; 111: 6452-6454.

45. Palmans ARA and Meijer EW. Angew. Chem., Int. Ed. 2007; 46: 8948-8968.

46. Gottarelli G, Lena S, Masiero S, Pieraccini S and Spada GP. Chirality 2008; 20: 471-485.

47. Pescitelli G, Di Bari L and Berova N. Chem. Soc. Rev. 2014; 43: 5211-5233.

48. Pale V, Nikkonen T, Vapaavuori J, Kostianien M, Kavakka J, Selin J, Tittonen I and Helaja J. J. Mater. Chem. C 2013; 1: 2166-2173.

49. Štěpánek M, Matějíček P, Humpolíčková J, Havránková J, Podhájecká K, Š́pírková M, Tuzar Z, Tsitsilianis C and Procházka K. Polymer 2005; 46: 10493-10505.

50. Fan H and Jin Z, Soft Matter 2014; 10: 2848-2855.

51. Connal LA, Lynd NA, Robb MJ, See KA, Jang SG, Spruell JM and Hawker CJ. Chem. Mater. 2012; 24: 4036-4042.

52. Li J, An Y, Chen X, Xiong DA, Li Y, Huang N and Shi L. Macromol. Rapid Commun. 2008; 29: 214-218.

53. Yao L, Lu X, Chen S and Watkins JJ. Macromolecules 2014; 47: 6547-6553.

54. Robb MJ, Connal LA, Lee BF, Lynd NA and Hawker CJ. Polym. Chem. 2012; 3: 1618-1628.

55. Krogstad DV, Lynd NA, Choi SH, Spruell JM, Hawker CJ, Kramer EJ and Tirrell MV. Macromolecules 2013; 46: 1512-1518.

56. Klinger D, Robb MJ, Spruell JM, Lynd NA, Hawker CJ and Connal LA. Polym. Chem. 2013; 4: 5038-5042. 
57. Bharatiya B, Schumers JM, Poggi E and Gohy JF. Polymers 2013; 5: 679-695.

58. Nunes SP, Karunakaran M, Pradeep N, Behzad AR, Hooghan B, Sougrat R, He H and Peinemann KV. Langmuir 2011; 27: 10184-10190.

59. Ogi S, Sugiyasu K, Manna S, Samitsu S and Takeuchi M. Nat. Chem. 2014; 6: 188-195.
60. Mikhalitsyna EA, Tyurin VS, Nefedov SE, Syrbu SA, Semeikin AS, Koifman OI and Beletskaya IP. Eur. J. Inorg. Chem. 2012; 12: 5979-5990.

61. Snitka V, Rackaitis M and Rodaite R. Sen tors, B 2005; 109: 159-166. 